phy is often due to the presence of cystic degenera. tion of these follicles; a pathological condition which often becomes much exaggerated when any of these diseased glands have been rolled into the cervical canal by trachelorrhaphy. Oftentimes the cysts are present in great numbers, and of such enormous size as to lead to the suspicion that the consequent enlargement of the cervix may be from malignant disease. Such a case has recently come under my observation, the patient having suffered from pronounced catalepsy since trachelorrhaphy was performed ten years ago. An examination showed atresia at the os externum, excessive enlargement of the cervix, right ovary partly fixed, size of English walnut, left ovary fixed, slightly enlarged. In order to ascertain the true condition of the interior of the cervix deep bi-lateral incisions were made, and the anterior and posterior lips, were spread wide apart as they are in the most extensive laceration. Much pent up secretion escaped from the uterine cavity, showing that the tight closure of the os externum had converted that cavity into a retention cyst; large numbers of cysts of the nabothian follicles appeared in the cervix, some miliary, others as large as small marbles. In the excision of these cysts, almost all the mucous membrane of the cervix was removed. The margin of the mucous membrane of the vaginal portion was then stitched with catgut sutures to the margin of the intra-cervical mucous membrane, both anteriorly and posteriorly, so as to fold the anterior and posterior lips of the cervix upon themselves. The lateral incisions were then closed with silkworm gut sutures, leaving the cervical canal perma. nently patulous. This operation was practically that of Schroeder. The perineum which was lightly lacerated was also closed. The operation was performed about six months ago, and the patient although naturally neurotic, reports herself free from cataleptiform seizures, since her recovery from the operation. This case, although striking, is only one of a large and increasing number of parallel cases, which illustrate possible evil results of bad surgery.

In the preparatory treatment for trachelorrhaphy, experience has shown that the simpie puncturing of cysts of the nabothian follicles by means of the spear pointed lance is inadequate, because the cysts are prone to be refilled. They should either be destroyed by the cautery or excised. The excision may be of the individual cysts, provided they are not too numerous, otherwise of the cervical mucosa.

The evil results consequent upon the abuse of Emmet's operation, are frequently encountered, and a large part of the most necessary work, in connection with this operation, at the present time, consists of the conservative surgical procedures already outlined, the object of which is to undo work which has already been done. If the abuse of the operation has done much harm, the misguided practitioner must bear the burden of his owl fault; the operation must not be made to do so for him.

IrNaR CAYsTIC.- "You are worth a great deal more to people at some times than you are at others," said the comet sneeringly to the moon. "That's why they call you the silver moon."

Yes," replied the luminary, sadly ; "it all comes of having to be taken at my phase value."-Washington Star.

\section{ORIGIN AND DEVELOPMENT OF THE GENITO-} URINARY ORGANS IN WOMEN.

Read in the Section of Obstetries and Diseases of Women, at the Forty fourth Ammual Jleetiug of the American MLedical Association.

\section{BY F. BYRON ROBINSON, B.S., M.D.}

PROFESSOR OF GY NECOLOGY IN CHICAGO POST-GRADUATE MEDICAL SCHOOL YNECOLOGIST TO WOMAX'S HOSPITAL, TO POST-GRADUATE HOSPITAL. TO CHARITY HOSPITAL AND TO COLUMBIA DISPEASARY.

The following remarks are based on original investigation in the human and in the lower animals, especially the pig. The research embraces the examination of about 1,000 specimens of the genitals of the human and lower animals in various conditions and stages of development. The investigation was carried to the bird family, and some interesting information was gained. The development of the genito-urinary organs is one of the most fascinating of all studies, as the plans of evolution are most perfectly manifested in rapid succession. As the organs develop, the subjects of hypertrophy and atrophy are seen in their most typical forms. It is plain that in the embryo whole ages of evolutionary development take place in a few weeks, and that each of the distinct stages of the then distinct kidneys of mammals represent so many wide periods of animal life. The economy and conservatism of nature is shown in its most beautiful mauner-in the development of the genito-urinary organs by inducing organs and ducts to act first for kidneys and excretory organs, and finally for egg channels and gestation sacs. The great capacity that organs have to modify themselves is a most instructive subject. Every young physician should acquaint himself with the embryological development of the part of anatomy to which his future practice is likely to lead. Embryology aids in comprehending the history of the growth of the genitals. It enables one to comprehend the adult genital organs and gives frequently a clue to their pathology. The so-called practical man may attempt to announce that the study of the development of the genitals is worthless because we can not change the course of deficient nature. But to the scientific physician every stage of the development of the genito-urinary organs is an incentive for hopeful acquisitions that may avert deformities. Malformation of the female genitals is simply nature caught in the act of wrong development. In the widespread awakening of the present it is interesting to observe the family physician attempting to study the female genitals so that he may understand arrest of development, relics of atrophied organs and tumors from the same. To be practical one must follow every stage of developmental structure. Comparative anatomy sheds a flood of light on such work, and if one will carefully investigate the grow th of the Wolffian body among the lower animals and man, the adult form of the genitourinary organs will be relatively easy of comprehension. About 1750, Dr. Wolff of Berlin, Germany, begain to announce the results of his studies on the genito-urinary bodies. The subject was so attractive that it drew to it some of the most brilliant names of literature who have enriched our libraries. Mueller, Gartner, Rosenmuller, Waldeyer, Lankester, Raztke and Gegenbaur are but a few who have recorded their views on the Wolffian body. The magnificent labors of Balfour, which read like a bewitching story, aided by the industrious Semper, have enabled us to distinguish the three grand divisions of the Wolffian 
body. The best and most practical method of obtaining clear knowledge of the Wollfian body is to secure fetal pigs at the slaughter house. The development of the Wolffian body is similar in all mammals, and pigs can be obtained from a day old up to birth.

The Wolffian body is a complex, varying organ extending from diaphragm to cloaca or rectum on each side of the backbone. It is oval or spindleshaped. In a small fetus the Wolffian body is the longest visceral organ and almost fills the abdominal cavity. The dominating fetal viscus is the liver, which rapidly enlarges and induces the large and prominent abdomen of early vertebral life so characteristic of infants. As the liver enlarges the Wolffian bodies decrease, perhaps in accord with the view that only one viscus can induce a high blood supply. Under a good lens one can easily see the longitudinal ducts of the Wolffian body, and also the very numerous short ducts that enter at right angles to the longitudinal ones. In typical specimens the naked eye detects all this and much more. I will note the study of the Wolffian body in three stages, as it occurs in nature:

1. The first stage includes the head kidney and its duct or ureter. It is called the first kidney or pronephros, and its duct is the Müllerian duct.

2. The second stage is known as the middle kidney and its duct. It is called mesonephros, and its duct is known as the Wolffian duct.

3. The third stage is called the third kidney with its duct. It is the kidney of adult life and its duct is the ureter of adult life.

1. The head kidney or pronephros will not be given much attention, as it is not functionally in the higher vertebrates. It may only be active in the fetal life of man for a few hours. But in lower animals, as fishes, it functionates a whole fetal and adult life. It is the duct of the pronephros which concerns us as gynecologists, for it finally forms the vagina, uterus and Fallopian tube. The duct of the head kidney is known as Müller's duct. It is the Fallopian tube. It first carried urine from the head kidney, but it finally carried eggs. Nature is so conservative that she makes one organ do several functions. In the female, then, the duct of the first kidney carried urine and then eggs, while in the male it first carried urine and finally atrophies to a small cyst found on the testicle and known as the hydatid of Morgagni. Müller's duct of each side comes together in its posterior or lower two-thirds, and the approximated walls fuse so that one tube results. The fusion of the two ducts begins about the center of the middle third and proceeds both forward and backward. This peculiar method of fusion explains why we may have a single uterus and a double vagina, or a single vagina and a double uterus. The manner of fusion explains why we may have no uterus and a double or single vagina, or a uterus and no vagina.

The upper third of the Müllerian duct develops into the Fallopian tube. At the upper or anterior end of Müller's duct we can find, in some 15 to 20 per cent of cases, a small pedunculated cyst known as the hydatid of Morgagni. This hydatid of Morgagni is perhaps the drawn out end of the upper mouth of the duct of Müller, or it may be a dilated uriniferous tubule of the pronephros. In the chicken the duct of Müller forms a single channel from ovary to cloaca, and the funnel-shaped abdominal end of the
Fallopian tube opens out on the ovary; i. e., the rich hen's ovary sits right in the funneled mouth of the tube. In the hon, Müller's duct is merely a channel to discharge eggs, and it hardens or calcifies them as. they pass the irregularly lumened and tortuous duct. The Müller ducts in a chicken do not coalesce, so that it acts in the hen as a vagina, uterus and Fallopian tube while it is only one channel. But the hen's duct is lined with peculiar epithelium, and secretes albumen to coat the egg and calcium salts to protect it with a shell while the egg passes the duct, which lasts from one to three days. A hen has, curiously, only one Müller's duct and one ovary-the left. The right has atrophied. Dr. LucyWaite reported to me that while investigating the genitals of hens, she found remnants of the right atrophied Müiler's duct in the form of dilated cysts near the cloaca. I had also found similar vestigial remains of the atrophied right Müllerian duct in hens. At first I thought it was unique in birds to have one Müller's duct atrophied and only one duct and ovary functionating: but on further investigation it was found that several of the lower species of animals had the same nonsymmetry of ovary and Müllerian duct.

The explanation of the bird's loss of the right ovary and ova duct is not yet given. A very interesting subject was found in the varying upper or abdominal end of Müller's duct. In the pig, Müller's duct ends in an enormously wide, fringed funnel. This large funnel opened into a spacious peritoneal pouch in which lies the pig's ovary. In the ovulation of the sow the ova drop either, first, into this large peritoneal pouch and then into the funneled mouth of Müller's duct, or the ova drop directly into the mouth of the duct. In either case when the sow ovulates it is hardly possible to lose any ova on account of the peritoneal pouch. In animals with multiple births nature provides large means to secure rich supplies for reproduction through the peritoneal pouch and the peculiar ending of Müller's duct. There is a peritoneal pouch in many animals -e.g., cat, dog, mouse, pig-in which the fimbriated end of the Fallopian tube opens. The pouch is peculiar in each animal. The peritoneal pouch decreases in animals up to man. There is a small trace of a peritoneal pouch in woman which I have several times observed. In all animals which $I$ have investigated - sheep, dog, bird, cow, monkey, cat, man, there is a band of muscular or ligamentous structure which connects the fimbriated end of Müller's duct to the ovary. Ascending in the scale of animal life, we have, first, the eggs simply drop out of a hole in the peritoneum; second, the peritoneal pouch exists; third, the mouth of the duct surrounds the ovary, as in birds; and finally, the ligamentum ovaricum persists as it is in monkey and man. This little strip of muscular tissue which connects the mouth of Müller's duct to the ovary, shortens at menstruation or oestrus in all animals which I have examined. In shortening it draws the mouth of the duct over the surface of the ovary, and that part of the ovarian surface which is ovulating may emit an ovum into the duct's mouth; but if the ovulating surface of the ovary lies outside of the mouth of the duct, the ovum may be lost. In the cow as well as in the living woman, I have seen the wide mouth of the tube cemented to the ovary at its circumference by sticky mucus at menstruation. The disappearance of the peritoneal pouch is in direct ratio to 
the disappearance of multiple births. The typical sample of the peritoneal pouch at the end of the tube is the pig, which has a typical multiple birth. In man the peritoneal pouch at the tubal end is least, and typical single births characterize the genus homo. So that multiple and single births have some relation to the connection of the ovary to the mouth of the tube. Müller's duct varies widely in its form and coalescence from the lower animals to man. In some the two ducts do not coalesce; e.g., birds; in some they coalesce but a little; e.g., pig, cow and horse; in monkey and man we note that Müller's ducts coalesce for their lower two-thirds; i. e., up to the Fallopian tube.

One very valuable landmark to distinguish the uterus from the Fallopian tube was given to us by the immortal Virchow; that is, the round ligament. The round ligament inserts itself in the duct of Müller at the junction of the uterus and Fallopian tube. This means that all gestation is done in that part of Müller's duct posterior to or below the insertion of the round ligament. Gestation is never done in the Fallopian tube of animals but in the horns of the uterus. In a long search I have never known an authenticated case of tubal pregnancy in any animal below man. Only, so far, has it been demonstrated that in woman tubal pregnancy occurs. It would appear that a monkey was just as liable to tubal pregnancy as woman, from the structure and shape of the genitals, but no proof is yet recorded of tubal pregnancy in monkeys. It would be interesting to note the various kinds of placenta that the Müller duct bears, but space forbids. Suffice it to say that in the carnivora it is zonular; i. e., it reaches all around the circumference of the duct or gestation sac in the form of a band. In the herbivora the placenta is cotyledonous; i. e., the placenta rests on peculiar elevations of the gestation sac, shaped just like a mushroom on its stalk. In the omnivorous; e.g., the pig, the placenta is universal; i. e., the choroidal membrane lies close against the mucous membrane of Müller's duct or the gestation sac. In the quadrumana, as in monkey and man, the placenta is discoidal; i. e., the part of chorionic membrane which nourishes the child lies on the mucous membrane in the form of a disc. Müller's duct acquires various kinds of epithelium for a lining through the animal kingdom. Where the ducts coalesce into vagina, uterus and Fallopian tube as in most higher vertebrates there are generally three kinds of epithelium membrane, as, e. g., squamous for the vagina; glandular, columnar and ciliated for the uterus; and glandular, columnar and ciliated for the Fallopian tube. But whether the duct coalesces or not, a certain part of its epithelial lining possesses peculiar properties of secretory powers. In the bird the endothelium can secrete albumen and calcium salts. In mammals it possesses the power to gestate. In general, the ova may be viewed as a gland and the Müller's tube as its duct.

2. The second stage of the Wolffian body is known as the middle kidney or mesonephros. Its duct is known as the segmental duct or Wolffian duct. The Wolffian duct and the Müllerian duct appear to me to beformed side by side. Others assert that the segmental duct splits into two and thus is formed Mül. ler's and the Wolffian duct. The middle kidney or mesonephros and its duct probably functionates in the human fetus for six weeks. In the pig the mesonephros functionates for about one-half its fetal life. The middle kidney is the Wolffian body, proper, of vertebrates and it grows to a very large size. It consists of an elongated body lying on each side of the vertebral column and reaching from diaphragm to rectum or cloaca. One can easily see its long white duct running from its anterior end to its posterior end where it empties into the cloaca. At right angles to this long duct which collects the urine, may be seen dozens of short tubes running into the long tube. The chief portion of the Wolffian body is composed of these small coiled tubes. The duct of the Wolffian body first carries urine; finally it atrophies in woman and the functionless remnant is known as Gartner's duct. In the male it is transformed from a urinal duct to a channel to carry semen-the vas deferens. Nature is conservative and utilizes already formed structures for additional higher functions. Another feature is demonstrated, in that the urinary organs and genital organs are very intimate in their development and growth. For example, the kidneys, ovaries, tubes, uterus and vagina arise from the same Wolffian body; then their food, lymph and nerve supply must be absolutely connected. This principle of same nerve supply is applicable all through adult life and can be observed in many diseases; i. e., a diseased ovary will affect the kidney or a diseased tube will disturb a kidney, by reflex action through its circle of nerves.

The middle kidney or mesonephros, is of the highest interest to the gynecologist, because he has to deal with its vestigial remnants. In woman, the whole middle kidney atrophies and its residue is known as the parovarium. The remnants of this kidney were discovered by Dr. Rosenmuller in 1801. The name parovarium was given to it by Dr. Kobelt. The mesonephros has shrunk to a trapezoid shaped organ and lies in mesosalpynx; i. e., between the ovary and Fallopian tube. Its pointed or smaller end runs to the lumen of the ovary. It is composed of from eight to thirty tubules which run in a vertical direction. These tubes are the uriniferous tubules of the mesonephros. The tubes all pursue a course at right angles to a longitudinal tube known as Gartner's duct-the original ureter of the mesonephros. The parovarium consists of three quite distinct parts: (a) Gartner's duct. This runs toward the uterus and in cows it runs near the mouth of the urethra, occasionally. It no doubt represents Skene's tubes. In the pig it is often many feet long and as large as a clay pipestem. It has a large lumen in the pig, and I have frequently noted its atrophy at different intervals of its course, so that it represents beads strung on a string. It shows also interrupted atrophy in the woman. I have several times, in laparotomy, seen Gartner's duct dilated; once to the size of a child's head, and again I saw it dilated as large as an apple and the dilatations were strung along like beads. Perhaps each dilatation represented the entrance of a uriniferous tube.

$b$. The chief remnant of the mesonephros is the vertical tubules of the parovarium, varying from five to thirty, visible to the naked eye. To see the tubules one should hold the broad ligament up to the light. These little tubules frequently dilate into cysts from the size of pin heads up to that of a child's head. The tubules have a lumen lined with epithelium.

$c$. The third structure found in the remnant of the atrophic mesonephros is that known as Keibel's tubes. 
These are flask-shaped cysts or dilated parts of the uriniferous tubules situated external to the vertical tubules. They frequently dilate and gradually acquire a long, thin pedicle. The reason of the existence of these blind, flask-shaped tubes of Keibel must be the same as that for the hydatid of Morgagni.

$d$. Besides these three distinct structures, we find other relics lying in the broad ligament in the form of isolated cysts lying under the peritoneum. But they have definite locations, e.g., some are nearly always found at the upper border of the abdominal tubal sphincter. Another place is along the tubal fimbriæ which connect the tube to the ovary. They are all remnants of the mesonephros. The parovarium has pathological cysts in it in 60 per cent. of women according to my examinations. I have per- formed laparotomy for parovarian cysts on women from 23 years up to 55 years. Parovarian cysts do not develop before puberty, but they may develop as long as the woman lives after puberty. The repeated rupture and refilling of parovarian cysts $I$ have noted in my own patients. No peritonitis occurred. Sows have parovarian cystic degeneration. One distinguishes a parovarian cyst from other cysts by its capacity of being peeled out of the broad ligament.

3 . The third stage of the genito-urinary bodies is known as the metonephros or adult kidney. Its duct is the ureter. The last kidney grows out just behind the mesonephros. It starts as a little white body, and soon grows to a large size. It has at first a spiral, crooked ureter, but as the bladder sinks down into the pelvis it straightens out. The atrophy of the middle kidney and the hypertrophy of the third is a fascinating subject. The blood stream is simply turned from the middle kidney to the last kidney; the one shrinks and the other grows. The mesonephros simply pales from lessening blood; the metonephros reddens from excess of blood. The mesonephros atrophies and the metonephros hypertrophies just by changing the blood current. It may be observed that the duct or ureter of the first kidney persists in woman as Müller's duct, making the vagina, uterus and tube. In the male it is the duct or ureter of the second kidney that acts as the vas deferens and epididymis to carry semen.

By slitting open the abdomen of fetal pigs in various stages one can observe the beginning and course of the ovary. It arises from the internal sides of the Wolffian bodies as a white spot as large as the head of a pin. It is very vascular and very glandular. It grows rapidly from a kind of large, luxuriant epithelium heaped up into a ridge named the genital ridge. It is highly supplied by nerves and lymphatics. It does not manifest sex until the tenth week in the human, and acts similar in the lower animals. One can tell sex by the external genitals far sooner than by the testicle or ovary. The ovary soon after the first three months begins to manifest its Grafian follicles, and it is always seen to be a cystic organ. It sinks down in the pelvis and its artery, the ovarian, stretches out and becomes very lovg in the upright animals. The ovarian arteries arise from the aorta, just below the renal, at the original site of the growth of the ovaries. The Wolffian body is held to the diaphragm by a strong ligamentous cord which may be called the diaphragmatic ligament. The body is also fastened to the groin by a process which is surrounded by peritoneum like the ferrule on a fork handle. This process of tissue becomes the round ligament, while the peritoneum which surrounds it becomes the canal of Nuck. This process of round ligament formation with Nuck's canal can be observed to perfection in the grow th of fetal pigs. It may be observed that adult kidneys of many animals are lobulated; the reason of this is that the adult kidney arose first in a lobular or separate form. Each lobule was fed by an artèry, so that even in adult animals some kidneys may be found with several arteries. The kidney of a human fetus I have seen lobulated but it soon gets smooth. In man, dogs, pig, sheep, etc., the kidney is smooth, and in 75 per cent. it is fed by one renal artery. Among some fifty human cadavers which I have carefully dissected I found that 25 per cent. of the kidneys had two to five arteries. In the cow, one can see the typical lobulated kidney which must originally have had an artery for each lobule. The Wolffian body was originally generally drained by one vein and that would be the renal vein. But the upright position of man and monkey has induced the ovary to fall into the pelvis and this lengthened the ovarian vein. This ovarian vein originally opened into the main vein which would be the renal. At present the left ovarian vein opens at right angles into the renal vein and it represents the original fetal life. But the right ovarian vein opens at present into the vena cava just below the renal vein. Now the explanation I offer for this is that the evolutionary changes have occurred on the right side by the upright position, as then the ovary would drag on the ovarian vein and finally it would be apt to enter lower down in the long vena cava, while the left side still retains its original fetal condition. Gegenbaur explains it in another way by saying that the left ovarian vein is a remnant of the cardinal vein ; considerable disease is due to the difficulty of venous return on the left side. The supra-renal bodies, which develop with the genito-urinary, lie in the still unknown field of physiology. They have some relation with the sympathetic nerve whose significance is yet unknown.

1. Out of the Wolffian body arise the uterus, vagina and Fallopian tube. The ovary, parovarium, kidney and supra-renal bodies come from the same source. Hence they all have a physiological and anatomical connection.

2. The genital and urinary organs arise from the same source and occasionally one is modified to act for the other.

3. Since the whole of the genital and urinary organs have both an anatomical and physiological relation, the essential knowledge for the gynecologist will lie in the nerve connections, for disturbance in one organ will disturb the balance of all.

4. Injury to the nerves in one organ will disturb the circulation and nutrition in the other by reflex action.

5. The peritoneum and genitals are anatomically and physiologically connected. Hence disturbance in one will disturb the others.

6. The chief nerve supply of the genitals and urinary apparatus is sympathetic.

7. The main pathology of the sympathetic nerve is reflex action which will disturb any distant or adjacent viscus.

8. The irritation in pregnancy passes up the hypogastric plexus to the abdominal brain and is reflected out on the renal plexus, inducing albumen in the urine. The aching back in menstruation is accounted 
for by the close nervous connection of genitals and urinary organs. The renal plexus and the lateral ganglionic chain is dragged on by the hypogastric plexus.

9. The nephritis and albumen in the urine after vaginal hysterectomy is due to the wounding of the hypozotic plexus and ovarian plexus, and the irritation is reflected into the renal plexus disturbing the kidney. The shock from abdominal and vaginal section is due to the damage inflicted on the sympathetic plexus, and is often manifest in kidney disturbance.

10. The intimate anatomical and physiological connection of the genitals, kidneys and peritoneum by nerve, blood and lymph supply invites dangers to one by wounding the other. The danger of disturbed balance is brought about by reflex action flashed from one organ to another through a reflex arc.

11. The anterior and posterior columns of the vagina represent the remnants at the points where the ducts of Müller coalesce, producing thick columns of tissue.

\section{DOMESTIC CORRESPONDENCE.}

\section{Does Tuberculin Scatter Tuberculosis?}

AN OPEN LETTER TO DR. HEATWOLE OF GOSHEN, IND., FROM DR, DENISON OF DENVER, COL.

Dear Doctor:-The following are two extracts from the Goshen Daily News of Sept. 4, which some one has kindly sent to me. The cuttings are here transposed from their relative positions in the obituary notice, "A good citizen gone," so that the postmortem, which is what I wish to discuss, comes first :

"A Post MorteM.-This forenoon Dr. Heatwole conducted an autopsy on the body of the late George $X$. Thomas, assisted by Drs. Bowser Latta, Ash, Irwin, Miller, Johnson and Whitmer. It confirmed the diag-
nosis of the case, and revealed the fact that the Koch treatment had stopped the progress of the disease on the lungs, but had scattered it all over the glandular system, finally attacking the spine and bruin which was the immediate cuuse of death.

"The growth just under the knee proved to be a solid gland, five by seven inches, and had invaded the knee to such an extent as to entirely destroy the joint, the ends of the bones at the knee joint having entirely disappeared.

"He had been a great sufferer for nearly three years, and especially so for the past few months, which he bore bravely and hopefully, although recovery was hopeless. About seven years ago a growth like a tumor appeared on his leg just back of the knee, caused by tuberculosis. The growth increased gradually, but not until about three years ago were his lungs affected. Since that time he has traveled in climates which promised benefit, but improvement was only temporary. While in Denver he took the Koch treatmeut and it apparently healed his lungs, but the growth on his leg was constantly increasing in size until the leg became as large as his body and very painful. A few days ago tubereular meningitis developed, becoming so severe on Saturday that he became un conscious that night, in which condition he remained until his death."

Prompted by a desire to do justice to a most useful remed (tuberculin) I am persuaded to write you.

For it has seemed to me that we ought to blame ourselves more and the remedy less, and then there would be hope of our becoming well acquainted with tuberculin and its modus operandi,as well as that of the disease it antagonizes, to use it intelligently and with good results. The medical profession must be introspective and look to tuberculosis as well as to the composition of the remedy, for an explanation of the so-called "failures" of tuberculin. The delusion, which hinders a thorough understanding of the remedy, is that it inoculates with tuberculosis, or the above inference that it spreads the disease. Whenever insufficient diagnosis, unsuitable conditions for a cure, and excessive use of tuberculin, such as obtained in the unfavorable results in the Johns Hopkins and the Pennsylvania hospitals,--whenever these conditions are behind the so-called "failures" I feel like nailing the evidence where it belongs. This is my reason for wishing to review this case of my former patient, (Nov. 1892 to March 1893), to see if this dissemination of tuberculosis through the glandular system was subsequent to or followed the administration under my hands of Koch's tuberculin. On this point it seems to me there are fairly three reasons why the stated conclusion of the autopsy is in error. Of course I want to admit that this may be merely a lapsuspennx of a newspaper reporter, but it expresses a popular prejudice and a false position taken by many physicians inexperienced with this new method of treatment, which position is not easy to combat with our insutficient knowledge of tuberculosis and tuberculin. The three reasons are:

1. The tumor in the popliteal space antedated the appearance of any lung lesion whatever by three years. I deem it unnecessary to refer to the frequency with which tuberculosis originates in other parts before manifesting itself in the lungs. Of course there may be a question raised as to the fact, but I am going to assume that the nature of this tumor, as stated in the account, was unique all the time from its beginning; i. e., tuberculous, because under tuberculin treatment it grew hard and there was an effort to contract or shrink, and because the postmortem evidences showed that the most prolonged existence of tuberculosis was in the joint underneath the tumor. I had my good friend Dr. C. Theodore Williams of London, when he was visiting us last November, see this patient. When the doctor felt the tumor he exclaimed: "Oh! lymphadenoma; there is no question about it." All of which would go to show that tuberculosis was behind the lymphadenoma, and served to confirm in $\mathrm{my}$ own mind a theory I have had for some time; that the bacillus of tubercle is not the beginning of tuberculosis. Instance our general inability to find the bacillus in glandular tumors known to be tuberculous by tuberculin reaction or otherwise, or in adenoid growths in the region of the third tonsil which I have known to be removed, as simply adenoid, from those who were steadily advaneing in the tubercular process, as subsequent history proved.

2. The implication of the glandular system antedated the use of tuberculin in this case, and may have been a resultant of la grippe two years previous. I have known of other supposedly tuberculous subjects in whom the influenza seemed to center the tubercular poison in the glands of the neck. In this case both the thyroid glands were becoming much enlarged, there were nodular glands above the clavicles and both breasts were tender and as large as a young maiden's.

3. The reaction to tuberculin was not only in the affected lung, but in all these affected glands, especially in those above the clavicles, the breasts and in the tumor under the knee, showing that there was tubercular contamination there before tuberculin was given. In the case of the tumor on the Ieg, the size of which was $7 \frac{1}{2}$ inches, measured lengthwise with the limb, and $101 / 2$ inches across the center, the effect of the treatment was a hardening of the soft mass and a restraint of its growth during the continuance of the tuberculin injections. The pain caused there was a positive hindrance to the use of the larger doses of tuberculin. This was the principal reason, probably, why Mr. Thomas gave up the method at two different times although in all other respects the effect was quite favorable. At one time he almost agreed to the proposition, which the autopsy now proves would probably have been best, namely, to amputate the limb and continue the immunity producing effect of tuberculin. It was with a view of settling this question that Mr. Thomas wished to consult with Dr. Senn, and I was glad to have him do so, but of course did not expect Dr. Senn to take my view of an operation with his expressed opposition to the use of tuberculin.

I think I have made out my case that in this instance the scattering to the glands had occurred before the tuberculin 